\title{
Clinical Nurse Specialist, Role Preparation, for Example, Educating the Educators
}

\section{Sheelagh Wickham *}

Memorial Hospital, Darlington, USA

*Corresponding author: Sheelagh Wickham, Emeritus Consultant Obstetrician, Memorial Hospital, Darlington, USA, Tel: 815.753.6584; E-mail: sheelagh.wickham@dcu.ie

Received date: November 11, 2014, Accepted date: November 19, 2014, Publication date: November 24, 2014

Copyright: @ 2014 Wickham S. This is an open-access article distributed under the terms of the Creative Commons Attribution License, which permits unrestricted use, distribution, and reproduction in any medium, provided the original author and source are credited.

\section{Editorial}

The role of the Clinical Nurse Specialist (CNS) has grown and developed over a number of years. Clinical Nurse Specialists are an increasing part of nursing practice in the twenty first century. Their roles are many and varied and indeed so are the benefits, particularly for the patient [1-3]. In the current constrained economic times there is an increasing awareness of the financial benefits of employing CNS to support patients. Other benefits also accrue, including increasing benefits in direct patient care, patient outcomes and job satisfaction for the CNS. However, it is not enough to research and record the benefits of the CNS. In the dynamic world of health care it is essential that practitioners, such as CNS are facilitated in preparation and ongoing life-long learning, particularly in their area of specialist practice.

To continue to see the growth and development of the CNS and the benefits to patients it is necessary to continue to support the CNS. However it is important to remember that support and development does not start after the appointment of the CNS but should ideally begin in the preparation for the post. Preparation for the role of the CNS varies. In most instances the CNS is expected to have studied or be currently studying to masters level at a minimum [4-6]. Such educational preparation is necessary considering the many and varied roles of the CNS such as, clinical specialist, educator, innovator, researcher etc. $[1,7,8]$. To date there is no single course or preparation for the CNS role and I would argue, considering the diversity of the CNS work, there could not be. However core skills such as research and education could be included in courses preparing nursing to practice at a specialist level. Currently it must be questioned where the skills needed by the CNS to practice in all the numerous roles originate from, research skills may be covered in a master's programme but are education skills?

Education not only plays a part in the preparation and ongoing development of the CNS it is recognized as an important role, this can be seen by the research in the many different specialist areas of practice $[9,10]$. Nevertheless, despite these various studies which recognise the importance of the educational role there is no clarity on what preparation the CNS require or obtains in order to function as an educationalist.

The educated patient is informed and empowered, and can, if $s /$ he wishes, actively participate in her/his care and decisions surrounding it. It may be argued that as CNS are just that, specialists, their depth of knowledge and experience can help contribute to their role as patient educators. However their ability to impart this specialist knowledge and educate does not appear to be an obvious part of CNS preparation. Education plays a large part in specialist practice. Education by the CNS is delivered in many areas such as their patients, their families and carers, colleagues and other healthcare workers. Yet how can a
CNS be expected to deliver education to the range of recipients without some training and preparation?

It is very difficult to find research exploring education elements of course content but an early and interesting study in the United Kingdom by Nolan, Nolan and Booth [11] evaluated course curricula and explored nurse preparedness for patient education in rehabilitation. Findings showed that overall nurses were ill prepared and identified that while the curricula for specialist courses reviewed did include "explicit references to a nursing role in patient and/or carer education" (2001, p.170) a lack of standardisation was highlighted. For example one course for specialist nurses had 92 contact hours in which to achieve 48 learning outcomes, a task that Nolan, Nolan and Booth (2001) understandably found hard to envisage. Unsurprisingly they questioned the preparedness of nurses to educate patients. However, it must be remembered that this was a review and did not explore the patient's or nurse's experience. Nevertheless it does illustrate the need for curricula to include education and training as part of CNS preparation.

As the literature shows a nurse needs to be knowledgeable and active in many roles to be considered a specialist. The role or skills required of the nurse working at a specialist level are highlighted in many articles. Preparation for such work is essential. The educator role of the CNS is a single but vital role. While, as acknowledged, there cannot be a single programme of preparation for the CNS role, there can be certain elements which should be included such as education skills, it is a core role and must be prepared for. Such preparation will further enhance the CNS and help to contribute to ongoing growth in the contribution the CNS can make to bettering patient care [12].

\section{References}

1. Edwards, Karen (2011) Role of the clinical nurse specialist in the multidisciplinary team. Cancer Nursing Practice, 10: 22-6.

2. McDonald, David (2012) Who is the clinical nurse specialist? Canadian Nurse, 108: 22-5.

3. Dolan, Vickie J. \& Cornish, Nancy E (2013) Urine Specimen Collection: How a Multidisciplinary Team Improved Patient Outcomes Using Best Practices. Urologic Nursing, Sep-Oct; 33 (5): 249-56.

4. Löfmark A, Mamhidir A (2010) Master's level in primary health care education -- students' and preceptors' perceptions and experiences of the alteration in the clinical areas. BMC Nursing, 9. (10p)

5. Begley C, Elliott N, Lalor J, Coyne I, Higgins A,Comiskey C (2013) Differences between clinical specialist and advanced practitioner clinical practice, leadership, and research roles, responsibilities, and perceived outcomes (the SCAPE study). Journal of Advanced Nursing, Jun; 69:1323-37.

6. Wickham, Sheelagh (2014) Impact of formal educational level on clinical specialist role activity. British Journal of Nursing, 23: 167-72. 
Citation: Wickham S (2014) Clinical Nurse Specialist, Role Preparation, for Example, Educating the Educators. J Nurs Care 3: e116. doi: 10.4172/2167-1168.1000e116

Page 2 of 2

7. Jokiniemi, Krista,Pietilä, Anna-Maija; Kylmä, Jari; Haatainen, Kaisa (2012) Advanced nursing roles: A systematic review. Nursing \& Health Sciences, 14: 421-31.

8. Wickham, Sheelagh (2013) What are the roles of clinical nurses and midwife specialists? British Journal of Nursing, 22: 867-75.

9. Whayman, Kathy, Stansfield, Cath, Duncan, Julie, OÂ Connor, Marian (2012) Developing the role of the clinical nurse specialist in inflammatory bowel disease Gastrointestinal Nursing 10: 22-7.
10. Gosden C, James J,Winocour P, Turner B, Walton C et al. (2009) Leading the way: Journal of Diabetes Nursing,; 13 (9): 330-2, 334, 336-7

11. Nolan J, Nolan, M, Booth A (2001) Developing the nurse's role in patient education: rehabilitation as a case example, International Journal of Nursing Studies 38:163-173

12. Roberts Jennifer, Floyd Sue, Thompson Shona (2011) The Clinical Nurse Specialist In New Zealand: How Is The Role Defined? Nursing Praxis in New Zealand, Jul; 27 (2): 24-34. 